\title{
Inverter generator sets for grids carrying heavy-load users
}

\author{
Pavel Ilyushin ${ }^{1 *}$, and Aleksandr Simonov ${ }^{2}$ \\ ${ }^{1}$ Energy Research Institute of Russian Academy of Sciences, Nagornaya St., 31, bld. 2, Moscow, Russia \\ ${ }^{2}$ RTSoft - Smart Grid LLC, Verkhnyaya Pervomayskaya St., 51, Moscow, Russia
}

\begin{abstract}
Inverter generator sets (GS) are increasingly common in the electricity industry. The paper dwells upon the core advantages and issues of integrating inverter generator sets that must be considered at the design stage to make informed decisions. The authors present examples of computing the electrical parameters of inverter generator sets in a variety of operating situations. The calculated electromechanical transients show inverter GS's are more efficient compared to conventional synchronous generators for grid nodes that are 'weakly connected' to the grid as well as for islanded operation. The paper further proves that automatic control systems of GS inverters can feasibly feature parameter control laws that conform to the general and additional requirements of the system, which in turn are based on the parameter calculations. It shows that a feasibility study is needed to decide whether to use inverter GS's.
\end{abstract}

\section{Introduction}

Analysis of international best practices reveals that distributed energy sources (DES) are mainly implemented as renewable energy facilities (REF), a steady trend that affects how energy systems operate and how regional economies can develop [1].

Russia's DES's mainly operate non-Russian gasturbine units (GTU), gas-piston units (GPU), and diesel generator sets (DGS). These units feature good quality, exceptional reliability, and efficiency, as well as longevity; they require very infrequent repairs and mean time before maintenance is long [2].

Inverter GS's have been on the rise in recent years. Inverters are used to connect direct-drive GTUs, wind farms (WF), and solar farms (SF), as well as electric energy storage (EES) units [3]. As power electronics evolves, inverters find ever broader use for the integration of heterogeneous distributed energy sources in grids carrying heavy-load users.

The electrotechnical market offers a broad range of inverters that vary in specifications and control algorithms; each unit has its integration and operation specifics.

To integrate inverter GS's in a heavy-load grid, one needs to provide effective parametric control for a variety of operating situations.

Such integration should not worsen the operating conditions of the electricity users in the grid; instead, it should seek to provide more reliable electricity delivery.

In order to make sound decisions on using inverter GS's, it is imperative to analyze the advantages and potential issues of their integration as well as the tricky parts of simulating, calculating, and analyzing the operating parameters of such units.

\section{Benefits of inverter GS's}

Since there are no standard guidelines on the use of inverter GS's, engineering design agencies develop custom solutions on a case-by-case basis, which might be problematic further down the road.

Integration of inverter GS's in grid nodes, whether 'strongly' or 'weakly' connected, offers a few benefits over conventional GS's:

- The GTU can use a gas-turbine engine (GTE) that features variable speed (frequency) in the optimal speed domain; besides, it will not need a reduction drive that would be necessary to connect the engine to a synchronous generator. Notably, GTEs are efficient at $>$ 5,000 rpm. Empirical data suggests that reduction drives are associated with lower GTE reliability and longer repairs [4],

- Phase loads are symmetrized, as synchronous generators are not able to sustain phase-asymmetric loads for long, and relay protection and automation (RPA) systems will disconnect the generator to prevent it from overheating and associated damage. This situation may arise when a DES-equipped area is islanded whilst still carrying a load [5-7],

- Protections are less likely to initiate DES disconnection due to close SCs or voltage sags to below $0.8 U_{\text {nom }}$ for over $0.2 \mathrm{~s}$, which would be critical for conventional GS's,

- Input voltage amplitude, phase, and frequency can be controlled with a far shorter delay $(\approx 1-3 \mathrm{~ms})$ compared to conventional GS's, which enables the automatic control system (ACS) to run a variety of control algorithms:

- absolute and relative limitation of active power to provide a hot reserve and room for control;

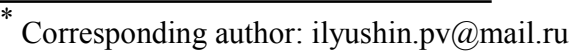


- limiting the rate at which active power changes, e.g. when the inverter is disconnected and reconnected;

- reactive power compensation by $Q(U)$ and $\cos \varphi(P)$

- active power adjustment for frequency, $P(f)$;

- active power limitation at higher voltage, $P(U)$;

- grid pickup at low and high frequency;

- grid pickup at low and high voltage;

- shedding active power and loading by reactive power [1],

- DES's including those based on renewable energy can be optimized for normal grid operation and efficient parameter stabilization in case of accident-related disturbances, i.e. for engagement in initial frequency regulation and voltage regulation. To that end, DES's based on renewable energy should contain EES units to smoothen the stochastic electricity generation patterns,

- The inverter output current can be limited to $\approx 1.2 I_{\text {nom }}$ in $20 \mathrm{~ms}$ to virtually nullify the effect on the $\mathrm{SC}$ current values in the grid. When connecting DES's, there is no need to replace high-voltage circuit breakers with new devices of higher switching capacity, nor there is a need to install additional current limiters. Besides, surge currents do not emerge in the case of an SC, thus, the GS-driving engine is not exposed to surges in electromagnetic torque,

- There is no need to stabilize the DES against external disturbances, which means there is no need to design for additional emergency control automations [8] including pole slip protections widely deployed at conventional GS's [9],

- Heterogeneous DES's can be integrated into hybrid energy facilities (HEF) using modular power converters to connect a variety of electricity users running on both AC and DC [10],

- No need for external synchronizers as synchronization is implemented in the inverter ACS software.

\section{Issues of inverter GS implementation}

Inverter GS's are integrated into low-voltage $(0.4 \mathrm{kV})$ and medium-voltage $(6-35 \mathrm{kV})$ grids. Engineers need to analyze the issues and make sound decisions when designing the connection for heavy-duty users; the design should be adjusted for the quality of the load. These are the issues:

- Unlike conventional GS's, inverter GS's lack the mechanical inertia of rotating masses, which, given how fast inverters are, can result in an instantaneous change in the power output in the first approximation. This does affect the rate at which the operating parameters will change in islanding-associated transients. Therefore, one needs to focus on configuring and parametrizing the inverters (manufacturer's defaults may result in GS disconnection) and to calculate the operating parameters appropriately so as to align the inverter control algorithms and protection settings with those of the grid RPAs [11, 12],

- It might be difficult to get RPAs up and running properly in terms of their sensitivity and selectivity due to little SC currents being fed to the damage site. When an inverter GS is islanded, i.e. providing all or most of the power, the local grid will need fundamentally novel RPAs that apply novel emergency detection algorithms [13],

- GS inverter protection settings might be difficult to align with the settings of standard RPAs in grids due to the low thermal inertia of integrated gate-commutated thyristors (IGCT) and insulated-gate bipolar thyristors (IGBT). Consider the following example of inverter protecting settings by current: $1.3-1.4 I_{\text {nom }}\left(t_{\text {trigger }}=10-100\right.$ $\mathrm{s}) ; 1.4-1.6 I_{\text {nom }}\left(t_{\text {trigger }}=0.1-10 \mathrm{~s}\right) ; 1.6-1.8 I_{\text {nom }}\left(t_{\text {trigger }}=0.1\right.$ $\mathrm{s})$ and $4.5 I_{\text {nom }}\left(t_{\text {trigger }} \leq 1 \mathrm{~ms}\right)$,

- The required power quality indicators (PQI) may be difficult to attain at the customers' busbars. Total harmonic distortion THDi is greatly dependent on the inverter load: the lower the load, the higher THDi, which becomes strikingly apparent in islanded operation. In DES's using renewable energy, EQPs are also affected by the stochastic electricity output, which in turn depends on the primary energy inflow. Significant PQI deviations limit the use of the existing digital instrumentations in RPAs, telemechanics, phasor measurements, etc. [14],

- Parameter maintenance might be challenging as manufacturers select inverter power values to save on the active power of the primary source. The PQ bar chart of the inverter shows that nominal active power is only provided at $\cos \varphi=1$, i.e. reactive power will not be provided without active power shedding. More powerful inverters should be used where feasible,

- Structurally and technologically appropriate use of inverters might be difficult to organize. To prevent unnecessary disconnections and damage, the following must be done before an inverter GS is launched:

- a full set of operating manuals must be available on site for the personnel to consult on how to respond to accidents and emergencies;

- maintenance personnel must be trained to handle inverters appropriately to minimize the risk of error.

Notably, some of the problems can be addressed by specifying strict requirements to inverters, while others can be resolved by adjusting the design and operation of electricity delivery to heavy-load users.

\section{Parameter analysis and calculation}

With respect to grids carrying inverter GS's, parameter calculation and analysis results are significantly affected by:

- The software that most design agencies use to calculate the steady states and transients. Such software usually lacks verified DES models including inverter GS models (direct-drive GTUs, WFs, SFs, and EES) that would be appropriately adjusted for the inverter algorithms, the technological protection settings, and the parameters of the primary energy sources (the driving engines),

- Software is unable to run harmonic analysis while monitoring the PQIs at the grid nodes, 
- Software is unable to run probabilistic analysis so as to calculate the parameters as affected by the stochastic electricity output of REFs.

The accuracy of parametric calculations depends on whether the equipment models are correct and the software is adequate to the problem setting. The results of these calculations determine whether the key decisions made when designing the grid will be right or wrong $[15,16]$.

Below is a real-world case where the engineers had to calculate the parameters for different options of integrating conventional and inverter generators in a grid.

For each option, the following integration methods were analyzed:

- Strong connection $\mathrm{X}_{\text {conn }}=2 \mathrm{Ohm}(\mathrm{L} 1=10 \mathrm{~km})$;

- Weak connection $X_{\text {conn }}=32 \mathrm{Ohm}(\mathrm{L} 2=150 \mathrm{~km})$.

All calculations including the electromechanical transients were run in PowerFactory, which has a large library of verified models: modern GS's, GS excitation systems, excitation controllers, primary drives (GTUs, GPUs, diesels, CCPPs, etc.), rectifiers, inverters, and asynchronous motors (AM). Motor load models are adjusted for the parameters of the rotated mechanisms.

The following disturbances were analyzed for calculations:

- A 3-phase SC near 110-kV busbars of the 110/10$\mathrm{kV}$ step-down substation see Figure 1, followed by the main protections disconnecting one of the two $110 \mathrm{kV}$ feeders $\left(t_{\text {disc }}=0.18 \mathrm{~s}\right)$, automatic reclosing not triggered,

- A heavy-duty AM starting on $10-\mathrm{kV}$ busbars.

For calculations, they monitored voltage at the $10-\mathrm{kV}$ busbars, voltage at the $110-\mathrm{kV}$ busbars of the $110 / 10-\mathrm{kV}$ step-down SS, the EMF angle of the synchronous generator, the AM rotation speed and the drive speed, the AM currents.

Figure 1 shows an integrated inverter GS.

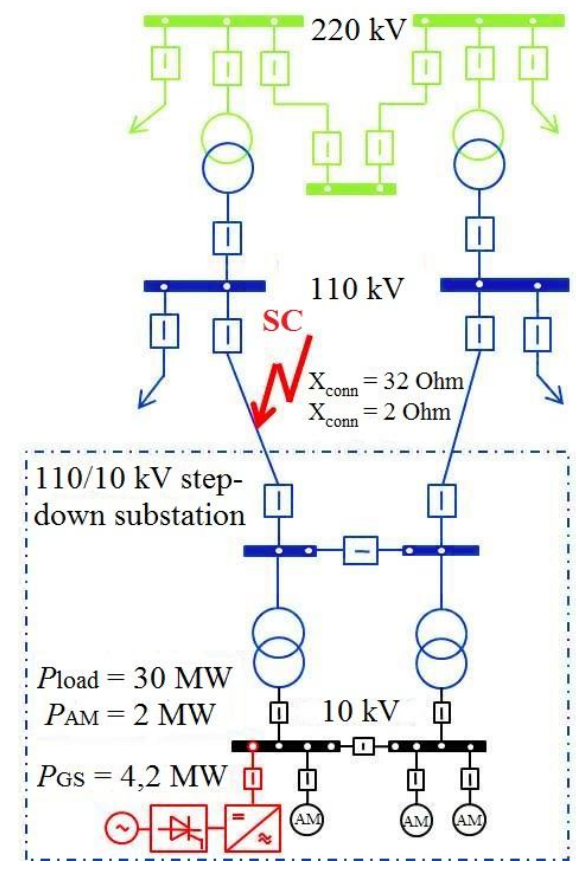

Fig. 1. Simplified single-line circuit of a grid segment.
Let us analyze the comparative calculations: conventional GS (SHUNT excitation and PMG excitation) vs inverter GS.

For strong connection and the disturbances specified above, calculations showed SHUNT / PMG coupled with a conventional GS to keep the GS and the loaded AM running stably. For inverter GS, both the current-based and the voltage-based GS control laws will keep the GS running, and the AM will remain stable.

In a 3-phase $\mathrm{SC}$ in a weakly connected grid, the conventional GS will be disconnected by the RPA as $U \leq$ $0.8 U_{\text {nom }}$ for $\Delta t>1.2 \mathrm{~s}$ for SHUNT, see Figure $2 a$ or $>0.8$ $\mathrm{s}$ for PMG due to the motor self-start. When using an inverter GS with control by the current, the voltage drops below the setting ( $U \leq 0,8 U_{\text {nom }}$ ) for $>1.0 \mathrm{~s}$, so there is a risk the protection will disable the GS. However, if the control law is voltage-based, the GS will keep running, see Figure $2 b$; the AM will remain stable (the $110-\mathrm{kV}$ busbar voltage of the 110/10 stepdown SS is shown in $\mathrm{red}$, the $10-\mathrm{kV}$ busbar voltage is shown in green).

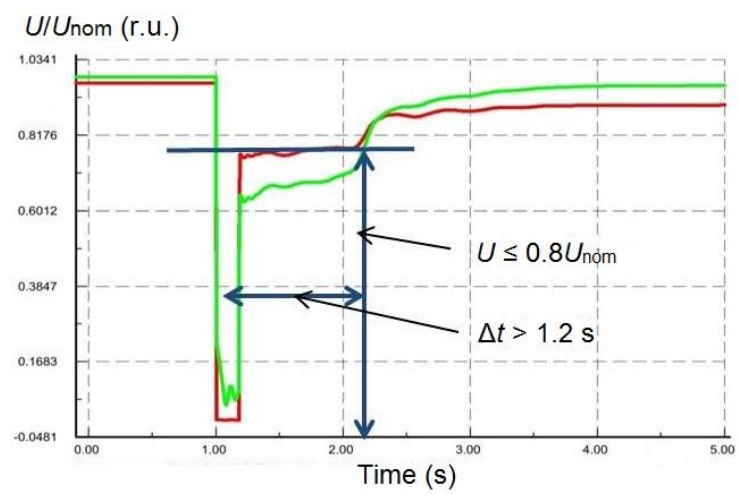

a)

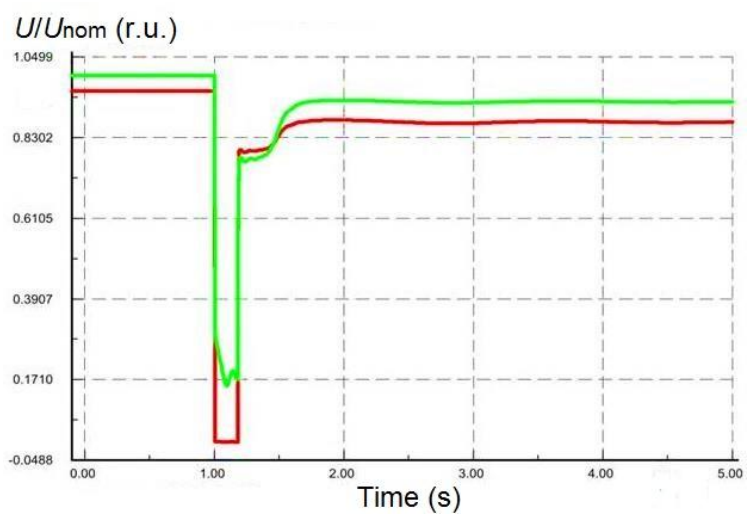

b)

Fig. 2. 3-phase SC transients: (a) for a SHUNT-equipped GS; (b) for a voltage-controlled inverter GS.

When starting a heavy-duty AM connected to $10-\mathrm{kV}$ busbars in a weakly connected grid, the conventional GS will be disconnected by the RPA since $U \leq 0.8 U_{\text {nom }}$ for $\Delta t>0.6 \mathrm{~s}$ for SHUNT, see Figure $3 a$ or $>0.4 \mathrm{~s}$ for PMG. When using an inverter GS with control by the current, the voltage drops below the setting for $>0.9 \mathrm{~s}$, so there is a risk the protection will disable the GS. However, if the control law is voltage-based, the GS will keep running, see Figure $3 b$; the AM will remain stable. 


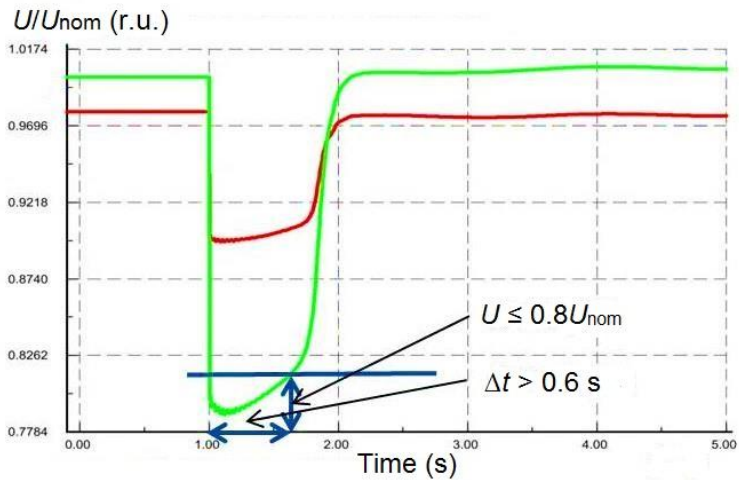

a)

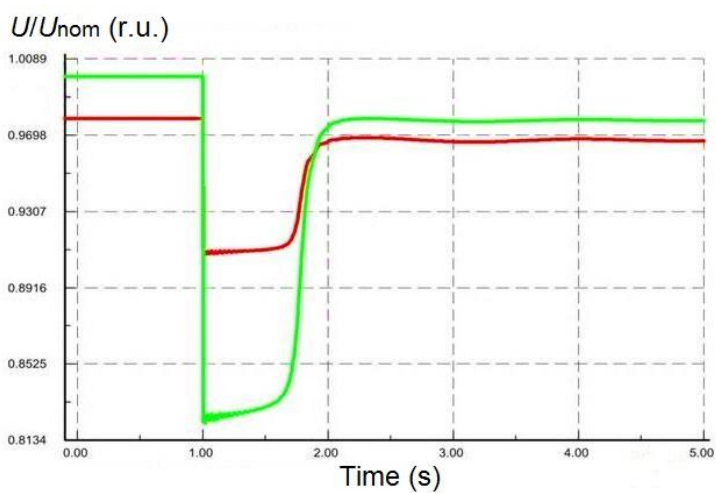

b)

Fig. 3. Transients caused by a heavy-duty AM starting on 10kV busbars: (a) for a SHUNT-equipped GS; (b) for a voltagecontrolled inverter GS.

Starting a heavy-duty AM on $10-\mathrm{kV}$ busbars in an islanded grid will cause the RPA to disconnect the conventional GS as $U \leq 0.8 U_{\text {nom }}$ for $\mathrm{t}>3.7 \mathrm{~s}$ for SHUNT, see Figure $4 a$ or $>1.4 \mathrm{~s}$ for PMG $[17,18]$. When using an inverter GS with control by the current, there is a risk the protection will disable the DES. When using voltage-based controls, the GS will keep running, see Figure $4 b$, and the direct motor start will be successfully completed.

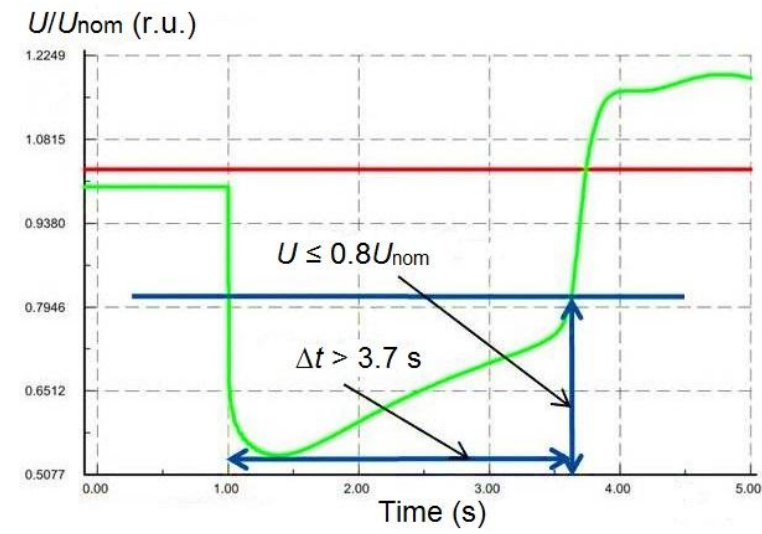

a)

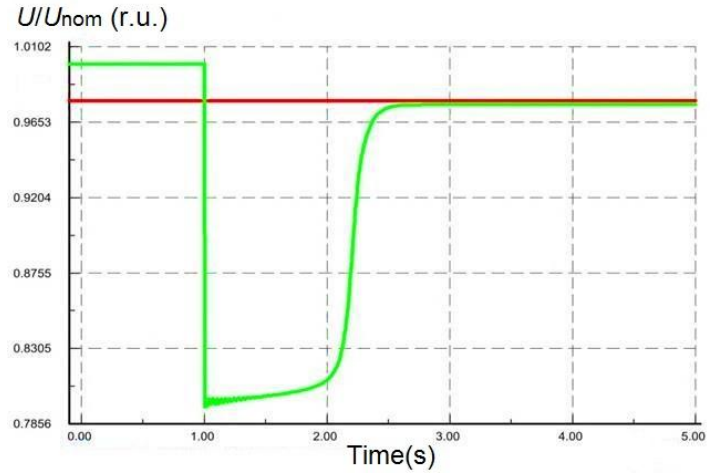

b)

Fig. 4. Transients caused by a heavy-duty AM starting on 10$\mathrm{kV}$ busbars in an islanded grid section: (a) for a SHUNTequipped GS; (b) for a voltage-controlled inverter GS.

The calculated electromechanical transients show inverter GS's are more efficient for grid nodes that are 'weakly connected' to the islanded grid compared to conventional generators.

However, appropriate inverter GS operation in islanded grids may require an additional controller (ACS reconfiguration) to implement a control function that will adjust the active power output to the frequency of the heavy-duty user's grid [19-20].

In light of the capital and operating costs, the decision to use or not to use inverter GS for a DES is subject to feasibility studies on a case-by-case basis.

\section{Conclusions}

As power electronics evolves, inverters find ever broader use for the integration of heterogeneous distributed energy sources in grids carrying heavy-load users.

Since an inverter can alter the output voltage amplitude, phase, and frequency very fast, it can implement the required control laws that should be selected based on general system requirements and additional requirements arising from the calculations of the operating parameters.

The inverter output current is normally limited to $1.2 I_{\text {nom }}$, which should be kept in mind when designing and configuring the relay protections for the grid.

Parameter calculations show that integrating an inverter GS in a strongly connected grid node will not affect its operations significantly; rather, its parameters will depend on the technical specifications and loads of the major synchronous generators in the adjacent grid.

Based on the results of calculations of electromechanical transients, the efficiency of using inverters for integrating DES into nodes that have "weak connections" with the power system, as well as in island mode, in comparison with the use of traditional synchronous generators is proved.

However, appropriate inverter GS operation in islanded grids may re-quire an additional controller (ACS reconfiguration) to implement a control function that will adjust the active power output to the frequency of the heavy-duty user's grid. 
The decision on the use of inverter switching at the DES GS facilities should be made during the design process on the basis of a feasibility study.

\section{References}

1. P. Ilyushin, A. Pazderin, R. Seit, Proceedings 17th International Ural Conference on AC Electric Drives, ACED (2018).

2. S. Filippov, M. Dilman, P. Ilyushin, Thermal Engineering, Vol. 66, 12, 869-880 (2019).

3. P. Ilyushin, P. Berezovskiy, Energetik, 11, 3-8 (2019).

4. V. Samoylenko, A. Pazderin, Promishlennaya energetika, 11, 31-35 (2014).

5. P. Ilyushin, Relay protection and automation, 2, 1224 (2018).

6. P. Ilyushin, Power Technology and Engineering, Vol. 51, 6, 713-718 (2018).

7. P. Ilyushin, K. Suslov, Proc. 2019 IEEE Milan PowerTech (2019).

8. S. Eroshenko, P. Ilyushin, Proc. 2018 IEEE 59th International Scientific Conference on Power and Electrical Engineering of Riga Technical University, RTUCON (2018).
9. P. Ilyushin, A. Mokeev, V. Narovlyanskii, Power Plant, 1, 52-59 (2019).

10. L. Rosman, Means and control systems in the energy sector, Overview information, Issue 2 (1987).

11. P. Ilyushin, A. Pazderin, Proc. International Ural Conference on Green Energy, UralCon (2018).

12. B. Buchholz, Z. Styczynski, Smart Grids fundamentals and technologies in electricity networks (Springer Heidelberg, 2014).

13. P. Ilyushin, Proc. of Methodological Problems in Reliability Study of Large Energy Systems, RSES, Vol. 58 (2018).

14. P. Ilyushin, A. Pazderin, Proc. 2018 International Conference on Industrial Engineering, Applications and Manufacturing, ICIEAM (2018).

15. P. Ilyushin, O. Sukhanov, Russian Electrical Engineering. Vol. 85. 3. 133-137 (2014).

16. P. Ilyushin, A. Kulikov, S. Filippov, Proc. Int. Russian Automation Conference (2019).

17. P. Ilyushin, A. Kulikov, Relay protection and automation, 3, 14-23 (2019).

18. A. Kulikov, P. Ilyushin, Electricity, 5, 4-11 (2019).

19. P. Ilyushin, A. Simonov, Relay protection and automation, 2, 30-38 (2020).

20. Sk. Islam, D. Sutanto, K. Muttaqi, IEEE Transactions on Power Systems, Vol. 30. 5. 25912603 (2015). 